\title{
Advanced Data Acquisition From Electron Microscopes With SerialEM
}

\author{
David N. Mastronarde ${ }^{1}$
}

1. Department of MCD Biology, University of Colorado, Boulder, USA

The SerialEM package provides a comprehensive interface for microscope control, image acquisition and display, and automated data acquisition of various kinds ${ }^{1}$. Although its main focus has been tilt series acquisition for electron tomography, it has also become widely used for acquisition of images for singleparticle reconstruction. Some of the key features and recent developments that have contributed to the program's popularity are briefly described below.

SerialEM supports all models of direct electron detectors from Gatan, Direct Electron (DE), and ThermoFischer Scientific (TFS) and provides control over the saving of movie frames. It now supports the latest generation of cameras, including the ability to control the electron counting provided in the TFS Falcon 3EC and in new camera server versions from DE. SerialEM also allows reliable alignment of frames, using the Framealign module shared with the IMOD program Alignframes. This module was first used to align K2 camera frames, and can now also be used with both Falcon 2 and 3 cameras, and with DE cameras run by the new server versions. Fig. 1A-D shows an example of images taken without and with frame alignment. Alignment time is variable, but for $\sim 4 \mathrm{~K}$ images, it is roughly $0.25 \mathrm{sec} / \mathrm{frame}$. For K2 and Falcon 2 cameras, it is slightly faster to align than to save the frames. Alignment during acquisition is particularly useful for tilt series, where shift alignment is sufficient.

The Navigator module in SerialEM allows one to save and return to positions, designate image files as "maps", and mark positions on those maps or other images. It provides high-level automation by moving to a series of positions and acquiring single images, montages or tilt series, or running a script at each position. A key component of this ability is a procedure for reliably returning to a position marked on a map, by re-imaging the area under the same conditions used to acquire the map and cross-correlating with the map.

The scripting capability in SerialEM has evolved to support quite complex operations. It has 360 commands that include: reporting on or setting microscope parameters; acquiring, manipulating, and saving images; and running many higher level operations in the program. The scripting language includes looping, conditional statements, arithmetic operations, some mathematical functions, variables (including arrays and variables that persist between script runs), and callable functions with arguments. One strength of the scripting is that new commands to access existing functionality in the program can be added relatively quickly to meet specific user needs. Several users have developed and shared scripts to manage all of the details of efficient, high-quality single-particle acquisition, which has facilitated the widespread adoption of SerialEM for this purpose.

SerialEM recently incorporated code adapted from $\mathrm{Ctffind} 4^{2}$ for fitting a contrast transfer function (CTF) to Thon rings in a power spectrum. There are two applications for this fitting. One is to give accurate estimates of defocus and astigmatism when the user clicks near the first zero of the CTF (e.g., Figs 1A, B) The second use is for new routines for accurate correction of astigmatism and coma based on fitted values of defocus and astigmatism. Fig 1E shows one of the beam-tilted images taken by the coma-free alignment routine. With a strong enough signal and stable conditions, the astigmatism can be corrected to within $\sim 10 \mathrm{~nm}$ and beam tilt to within $\sim 0.05 \mathrm{mrad}^{4}$. 


\section{References:}

[1] DN Mastronarde, J. Struct. Biol. 152 (2005), p. 36.

[2] A Rohou and N Grigorieff, J. Struct. Biol. 192 (2015), p. 216.

[3] Work on DE cameras was supported by Direct Electron, Inc.; I thank David Belnap and others at University of Utah for microscope access.
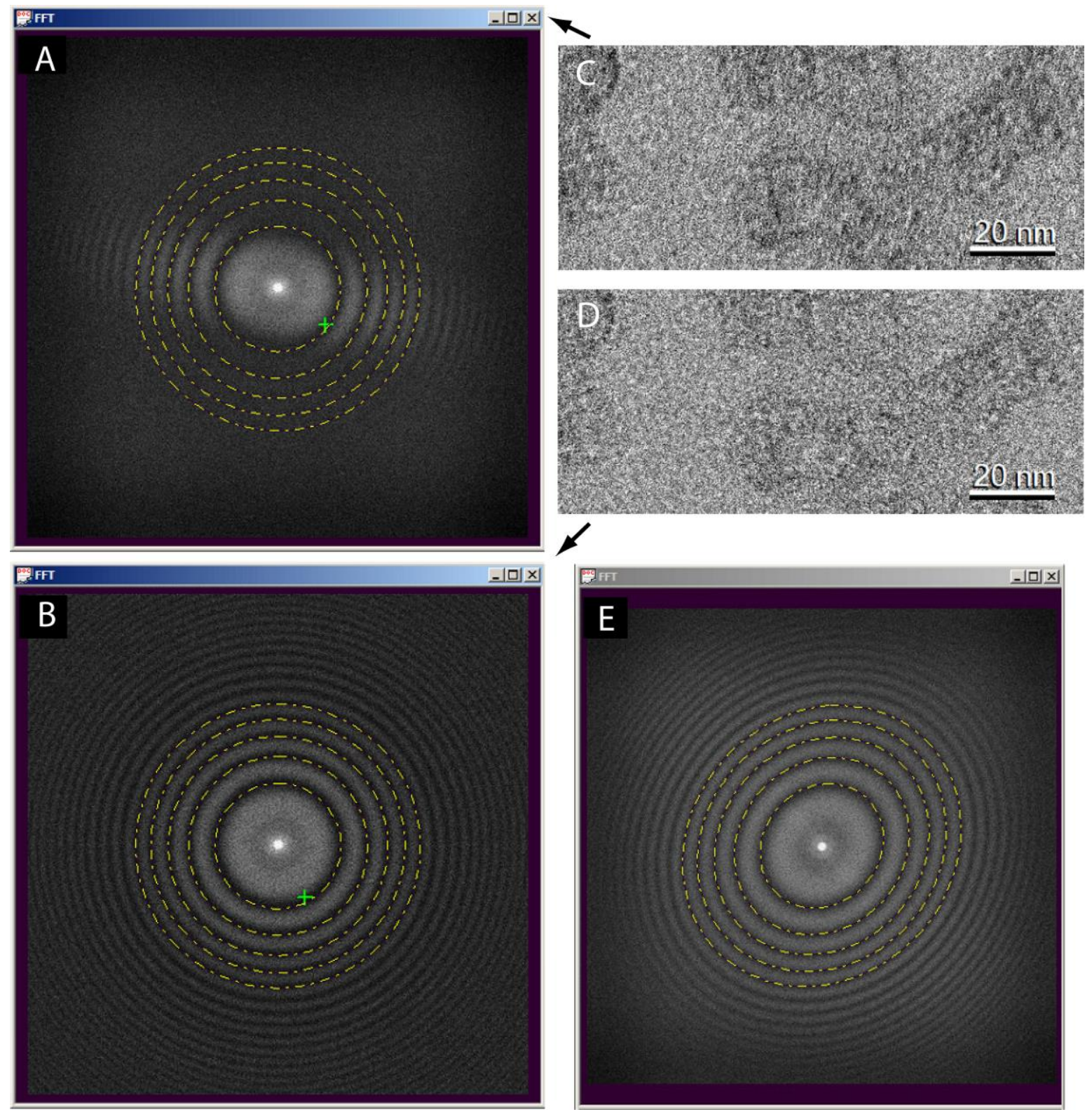

Figure 1. Illustration of the efficacy of frame alignment and of CTF fitting to astigmatic images. A, C. Power spectrum and subarea of corresponding image without frame alignment; B, D. spectrum and image with frame alignment. The sample is of ribosomes in negative stain imaged at $200 \mathrm{KV}$ on a K2 camera with a pixel size of $0.24 \mathrm{~nm}$. The $2 \mathrm{~s}$ exposure contained 20 frames of $0.1 \mathrm{~s}$ each; acquisition took $4 \mathrm{~s}$ without alignment and $9 \mathrm{~s}$ with it. E. Spectrum from image taken with 8 mrad beam tilt as part of the coma-free alignment procedure. In A and B, SerialEM drew the dashed rings after fitting initiated by clicking at the position of the green cross; in $\mathrm{E}$, it drew the rings as the procedure ran to show the fit. 